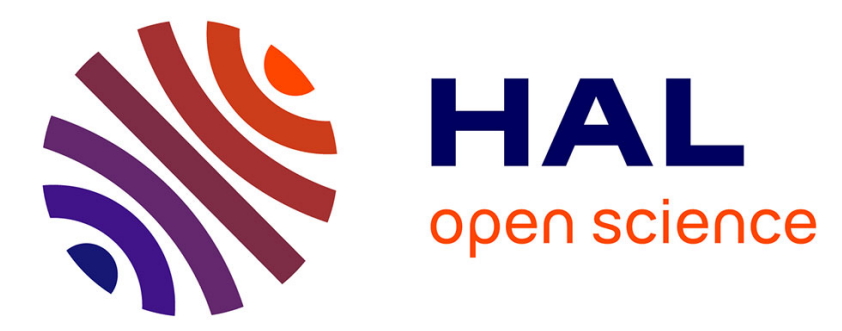

\title{
Développement de l'administration électronique par les communes françaises : éléments pour une typologie raisonnée
}

\author{
Amel Attour, Christian Longhi
}

\section{- To cite this version:}

Amel Attour, Christian Longhi. Développement de l'administration électronique par les communes françaises : éléments pour une typologie raisonnée. Géographie, Économie, Société, 2014, 16 (2014/1), pp.5-32. 10.3166/ges.16.5-32 . halshs-01062015

\section{HAL Id: halshs-01062015 \\ https://shs.hal.science/halshs-01062015}

Submitted on 9 Sep 2014

HAL is a multi-disciplinary open access archive for the deposit and dissemination of scientific research documents, whether they are published or not. The documents may come from teaching and research institutions in France or abroad, or from public or private research centers.
L'archive ouverte pluridisciplinaire HAL, est destinée au dépôt et à la diffusion de documents scientifiques de niveau recherche, publiés ou non, émanant des établissements d'enseignement et de recherche français ou étrangers, des laboratoires publics ou privés. 


\title{
Développement de l'administration électronique par les communes françaises : éléments pour une typologie raisonnée. ${ }^{1}$
}

\author{
Amel ATTOUR*, Christian LONGHI** \\ * Université Nice Sophia-Antipolis -GREDEG - CNRS - UMR 7321, associée BETA-CNRS-UMR 7522 \\ **Université Nice Sophia-Antipolis - GREDEG - CNRS - UMR 7321
}

Résumé : Cet article est une contribution à l'étude du développement de l'administration électronique locale dans les communes françaises. Après une revue de la littérature, il exploite une base de données originale pour mettre en évidence des différences importantes dans les stratégies de déploiement des services d'administration électronique locale d'un échantillon de communes homogène et l'importance des caractéristiques socio-économiques dans la compréhension de ces différences.

Mots clefs : Communes, administration électronique, TIC, Internet, territoire numérique, développement numérique.

\begin{abstract}
This paper is a contribution to the analysis of the development of e-administration in French municipalities. After a review of the literature, the paper exploits an original database and highlights important differences in the deployment of services of local e-administration of a sample of homogeneous municipalities, and the importance of the socioeconomic characteristics in understanding these differences.
\end{abstract}

Keywords: Municipalities, e-Administration, ICT, Internet, Digital Territories, Digital Development.

\footnotetext{
${ }^{1}$ Les auteurs remercient les rapporteurs anonymes de la Revue pour leurs remarques, critiques et suggestions, dont la pertinence a permis d'améliorer significativement la qualité de cet article. Les erreurs ou insuffisances pouvant subsister restent de la seule responsabilité des auteurs.
} 


\section{Introduction}

Les technologies de l'information et de la communication (TIC) et l'économie numérique sont profondément ancrées dans les territoires. L'utopie qui avec le développement de l'Internet anticipait la fin de la distance et de la géographie (Cairncross, 2001) s'est rapidement éteinte. «Never has a myth been easier to deflate. Not only do people remain highly concentrated, but the economy itself continues to concentrate in specific places. Place and community are more critical factors than ever before» (Florida, 2002, p. 219). La simple diminution des «coûts de transaction » associée à l'Internet a eu un effet désormais considéré comme classique par l'économie géographique depuis Krugman (1991), l'accroissement de la concentration des activités et des disparités des territoires. L'Internet a élargi le champ des activités considérées comme mobiles - jusque-là largement assimilées à l'industrie - et accentué les enjeux relatifs à la localisation.

Le développement local, le territoire comme projet de développement défini et porté par ses acteurs (les pôles de compétitivité ou les systèmes locaux de production en France par exemple), ont pris le pas sur l'aménagement par le haut, le simple déploiement d'infrastructures physiques (Longhi, Rainelli, 2010). On considère dans cet article qu'il en est de même pour les territoires numériques où le niveau local est de plus en plus important. Le déploiement d'infrastructures permettant l'accès au haut débit est certes un élément essentiel mais ce n'est pas, ou plus, le niveau fondamental (Musso, 2008, Marchandise, 2002, Attour et Longhi, 2009). A travers l'accès au haut débit, les investissements publics comme privés ont essentiellement cherché à résoudre le problème du «dernier kilomètre ». Mais dans la perspective du développement c'est « le premier kilomètre », celui où les usagers agissent et communiquent le plus qui est finalement le niveau à privilégier (Marchandise, 2002). Il ne s'agit plus de faire à distance mais d'offrir aux habitants comme aux entreprises et aux professionnels des services locaux performants (Bakis, 2010). 
Le déploiement d'infrastructures numériques, le type de technologie implémentée sur le territoire (Clark et al,. 2003 ; Gillett et al., 2004, 2006), ne peuvent être séparés de la nature des services qui y sont associés (Trkman et Turk, 2009). Trois dimensions interdépendantes doivent en effet impérativement être considérées pour qualifier la nature du développement local qu'il sous-tend: les infrastructures, les services et les usages (Longhi, 2007). En matière de développement des services d'administration électronique par exemple, l'absence de prise en compte des besoins des administrés semble expliquer la lente adoption et diffusion de ces services (Van Deursen et al., 2006 ; Wang et Liaw, 2008). Comme le soulignent Trkman et Turk (2009), la place accordée à l'administré utilisateur de ces services est en fait centrale. Plus largement encore, le déploiement des technologies d'accès et des services associés par les collectivités locales pose la question des stratégies de diffusion des services d'administration électronique mises en place par les acteurs publics locaux en vue d'en favoriser l'usage par les administrés ? Pour répondre à cette interrogation, il convient d'appréhender le développement de l'administration publique électronique locale en étroite relation avec celui des technologies de télécommunications (Trkman et Turk, 2009). Cette démarche méthodologique est d'autant plus importante que les communes ont très vite été reconnues comme un des acteurs locaux clefs dans le développement de l'administration électronique (Rallet et Rochelandet, 2004 ; Gillett et al., 2004, 2006).

En France par exemple, les villes petites et moyennes ont d'abord joué un rôle très actif dans le développement des infrastructures de télécommunication. Elles ont rapidement perçu les enjeux économiques et sociaux associés au haut et très haut débit, et ont beaucoup contribué en 2004 à l'adoption de la Loi sur la Confiance de l'Economie Numérique (LCEN, article L1425-1 du CGCT), qui donne aux collectivités locales la possibilité d'intervenir sur le marché des télécommunications et de déployer un réseau d'initiative publique en l'absence d'une offre privée. 
Les communes interviennent ensuite dans l'offre de services électroniques locaux, mais sont largement libres de la nature et du niveau des services offerts - hors quelques rares exception, il n'y a pas d'obligation légale faite aux communes sur le déploiement des services d'administration électronique - la diversité potentielle des développements à l'échelle locale rend l'analyse du niveau des communes pertinente. Leur implication tant au niveau des infrastructures que des services est par conséquent essentielle, elle fait partie de leur stratégie de développement local et du projet de territoire qu'elles portent.

Hormis quelques monographies et les travaux réalisés par le laboratoire M@rsouin ${ }^{2}$, qui constituent une exception, très peu d'études existent pourtant en France sur l'analyse du niveau d'investissements des communes dans le développement de l'administration électronique locale. Cet article est une contribution à la construction d'une base de connaissance sur la manière dont les communes françaises se sont saisies du développement numérique et des autorisations légales d'intervention qu'elles ont obtenues (autorisation d'intervenir sur le marché des infrastructures de télécommunications notamment) pour déployer l'administration électronique au sein de leur organisation.

L'article ne prétend cependant pas à l'exhaustivité. Il ne traite pas directement la diversité potentielle en matière de développement de l'administration électronique locale sous l'angle de la fracture numérique au sens où une large littérature 1'a déjà étudiée (Suire, 2003 ; Rallet et Ullmann, 2005 ; Lethiais et al. 2003 ; Dang Nguyen et Vicente, 2005 ; Vicente, 2005, Fautrero et al. 2007). Il n'aborde pas aussi les effets de l'aménagement numérique sur le développement local, au sens de son influence sur les performances économiques, où existe une importante littérature (Lehr et al. 2008 ; Crandall et Litan, 2008 ; Fornefeld et al. 2008 ; Atkinson et al. 2009; Katz et al., 2008; Katz et Suter, 2009; Lebenau et al. 2009 ; Koutroumpis, 2009). Il se cantonne à l'analyse des ressources (infrastructures et services

\footnotetext{
${ }^{2}$ Dang Nguyen et Trémenbert 2008, Dang Nguyen et al. 2011 par exemple.
} 
d'administration électronique) mises en place par les communes dans la perspective de favoriser le développement numérique de leur territoire, et vise essentiellement à la mise en évidence de stratégies différenciées dans la dématérialisation des services d'administration locale.

Plus précisément, il interroge l'existence de différentes classes - différentes stratégies ou politiques mises en œuvre - de communes et analyse leurs déterminants socio-économiques. Cela a nécessité la constitution d'une base de données originale - qui comble un vide dans l'information disponible et constitue en soi un premier apport. La méthodologie qui sous-tend la constitution de cette base - définitions d'un échantillon représentatif de communes et du questionnaire qui leur est administré - fait l'objet de la seconde partie de l'article. La première partie fait un état de la littérature sur le développement numérique et sur l'administration électronique, qui servira de base à la construction d'un cadre analytique adapté aux communes comme à la définition des informations pertinentes à assembler dans la base de données. La dernière partie analyse la base de données et met en évidence l'existence de différentes stratégies de développement de l'administration électronique locale adoptées par les communes, caractérisées par les déterminants socio-économiques de leur territoire.

\section{Comment appréhender l'offre de services d'administration}

\section{électronique locale?}

Pour caractériser l'implication des communes dans le développement des services d'administration électronique locale, cette première partie revient sur les facteurs qui ont contribué à souligner et élargir le rôle des acteurs publics locaux dans la numérisation de leur territoire. La littérature s'intéressant au rôle et à la place des acteurs publics locaux dans le développement des TIC sur leur territoire permet en effet de délimiter les différents choix et 
orientations possibles des initiatives publiques en matière de développement des services Internet au sein de leurs administrations.

Un territoire numérique se définit à travers une offre de services électroniques (limitée aux services locaux d'administration électronique dans cet article) associée aux infrastructures déployées sur le territoire physique. Ces deux dimensions ont mobilisé deux groupes de travaux. Le premier groupe s'est attaché à analyser le rôle et la nécessité d'une intervention publique sur le marché des technologies de télécommunication (1.1). Le second groupe s'est lui attaché à définir le concept d'administration électronique, ou plus généralement à analyser le concept d' «e-gouvernement» et à modéliser la trajectoire technique de son développement (1.2).

\subsection{Le rôle des communes dans le déploiement des technologies de télécommunication}

Comme le souligne la littérature, une raison fondamentale de l'élargissement des marges de manœuvre des collectivités locales sur le marché des télécommunications est essentiellement due à la libéralisation du secteur et à ses conséquences en matière de localisation de l'offre des opérateurs ${ }^{3}$. En effet, la structure de coûts des infrastructures et la nature des technologies d'accès existantes ont incontestablement conduit les opérateurs de télécommunication à concentrer leur offre dans les régions à forte demande potentielle (Vicente, 2005 ; Rallet et Ullmann, 2005). Cette concentration «de type réseaux métropolitains» (Dang Nguyen et Vicente, 2003) répondait essentiellement à une logique de rentabilité des investissements de la part des opérateurs de télécommunication. Rallet et Ullman (2005) rappellent, à l'instar de Hägerstrand (1993) et Lorentzon (1998), que l'innovation est introduite dans les centres économiques et se propage vers des espaces périphériques. Vicente et Suire (2004) constatent un renforcement des effets traditionnels d'agglomération et une croissance de plus en plus

\footnotetext{
${ }^{3}$ Pour une synthèse de la littérature, le lecteur pourra se reporter aux travaux de Vicente (2005).
} 
localisée sur les zones urbaines. Ils mettent en évidence un processus de localisation en cascade qui influence la concentration du marché des télécommunications en raison de l'existence de rendements croissant d'adoption. Cette concentration métropolitaine, observée partout dans les pays développés, en Europe comme aux Etats-Unis, persistait dans un contexte de mobilisation forte en faveur de la «Société de l'Information ». Elle a conduit les gouvernements à concevoir et mettre en œuvre des instruments de politiques efficaces pour remédier aux dysfonctionnements du marché. En France, ces actions ont amené les gouvernements à consacrer en juin 2004 les collectivités locales comme opérateurs possibles de télécommunications au sens de la LCEN. Les prérogatives de la LCEN attendent une forte mobilisation des investissements publics en matière de technologies d'accès, en particulier dans les régions rurales et périphériques (Dang Nguyen et Vicente, 2002). En effet, du point de vue théorique, les régions rurales sont caractérisées par une faible transmission des informations, un éloignement des partenaires, un marché du travail peu propice au déploiement de nouvelles technologies et par conséquent des infrastructures insuffisantes pour attirer l'implantation de nouvelles firmes (Galliano et Roux, 2006).

\subsection{Le développement de l'administration électronique locale : un modèle linéaire en plusieurs phases.}

Parallèlement à l'accentuation du rôle des acteurs publics locaux dans la diffusion des technologies d'accès, les chantiers de modernisation de l'Etat placent l'administration électronique parmi ses priorités (OCDE, 2004). Le développement des services d'administration électronique locale est en effet devenu un «impératif » pour la réforme de l'Etat dès le début des années 2000 (De La Coste, 2003) et plusieurs programmes gouvernementaux ont alors été lancés par la Commission Européenne. Au sein de ces 
programmes, les directives sont dans un premier temps essentiellement d'ordre technique ${ }^{4}$. Brousseau (2003), puis Dagiral (2007), soulignent ainsi que l'administration électronique a deux facettes. La première est de nature technique. Elle consiste à automatiser et à rendre accessible à distance et à travers la mise en ligne de procédures déjà existantes. La deuxième facette consiste en une réforme de l'administration publique via les TIC, susceptible de générer des transformations organisationnelles plus importantes que la première.

La première facette a mobilisé un grand nombre de travaux cherchant à modéliser la trajectoire du processus de dématérialisation d'un service ou d'une procédure administrative. Parmi ces travaux, le premier modèle de référence est celui de Layne et Lee (2001). Ce modèle appréhende le développement de l'administration électronique selon un processus évolutif en plusieurs phases. Plusieurs variantes ou extensions ont par la suite été proposées (Moon, 2002 ; Lee, 2003 ; Schelin, 2003 ; Reddick, 2004 ; Siau et Long, 2004 ; Ebrahim et al. 2004 ; Andersen et Henriksen, 2005 ; Saint-Amant, 2005 ; Van der Waal et Vloeimans, 2006). Dans ces modèles, la trajectoire de développement de l'administration électronique suit un processus en plusieurs phases où les trois premières caractérisent le niveau de l'informatisation des processus existants (phases «présence émergente», «présence affirmée » et «présence interactive ») tandis que les deux phases finales permettent une dématérialisation complète de la démarche administrative («présence transactionnelle » et « présence en réseau et intégrée »).

Ces modèles se rapprochent étroitement du modèle européen «e-Europe» appliqué depuis 2001 aux états membres, très vite généralisé à l'ensemble des pays du monde et adopté par de nombreuses études empiriques (Abanumy et al. (2005) pour l'Arabie Saoudite et Oman ; Limam Mansar (2006) pour Dubai ; Decman et al. (2003) pour la Slovénie ; Dijk et al. (2007)

\footnotetext{
${ }^{4}$ Pour une synthèse des différents programmes gouvernementaux et de leurs objectifs, voir Le Mentec (2011) « les fractures numériques sous l'angle des politiques publiques » in Plantard et al. (2011) « Pour en finir avec la fracture numérique », ed. fyp, pages $42-59$.
} 
pour l'administration hollandaise par exemple; Martin et Poussing, 2007 ; BacacheBeauvallet, 2011 pour la France). Le modèle «e-Europe »a en effet été fortement mobilisé par des travaux d'analyses comparatives du développement de l'administration électronique au niveau national quand le niveau 'local', pourtant fondamental, a longtemps été délaissé. Ce niveau local fait cependant l'objet de travaux récents (Jun et Weare, 2008 ; Dang Nguyen et Trémenbert 2008, Attour et Longhi, 2009 ; Dang Nguyen et al. 2011 ; Finney et Yoon, 2011 ; Lee et Berry, 2011, Dejean et Souquet, 2011). Il a nécessité, dans le cas français, un effort de construction d'une nomenclature des services d'administration électronique locale déployés ou potentiels (Attour-Oueslati et al. 2007, Dang Nguyen et Trémenbert, 2008 ; Dang Nguyen et al. 2011). Cette typologie s'est appuyée sur une double segmentation :

- Une première tient compte du destinataire des services comme le préconise SaintAmant (2005) dans ses travaux (les services « $G$ to $G^{5} »$ de commune à collectivités territoriales : procédures de dématérialisation des actes, relations internes, intranet ; les services « $G$ to $B^{6} »$ de commune vers les entreprises du territoire : dématérialisation des appels d'offre ; les services « $G$ to $C^{7} \gg$ de commune vers les habitants : services aux citoyens).

- Une seconde classifie les procédures administratives selon la nature du service (CapGemini, 2005). Elle distingue les services générateurs de revenus (impôts, taxes et contributions sociales, etc.), les services d'enregistrement (enregistrement d'un véhicule ou d'une nouvelle entreprise, changement de d'adresse, etc.), les services de redistribution (prestations sociales, recherche d'emploi, santé, bibliothèques publiques, etc.), enfin les permis et autorisations (permis de construire, passeports, inscriptions universitaires, etc.), les services dit de proximité pour recenser les services qui

\footnotetext{
${ }^{5}$ Government to Government.

${ }^{6}$ Government to Business.

${ }^{7}$ Government to Citizens.
} 
émanent directement des mairies et qui font l'objet de dématérialisation par initiative propre des mairies ${ }^{8}$.

L'offre d'administration électronique locale se mesure ainsi à travers l'état de dématérialisation de ces services. Il s'agit alors d'évaluer l'état de dématérialisation à travers un processus en plusieurs phases qui va de l'inexistence du service à sa mise en ligne complète sur le site web d'une mairie.

\section{Déterminants et adoption de l'administration électronique locale par les}

\section{communes}

Cette deuxième partie s'intéresse aux déterminants d'adoption de l'administration électronique par les communes. De manière générale, les facteurs d'adoption des TIC sont analysés par la littérature selon deux approches. La première se focalise sur les comportements d'adoption des usagers (internautes, administrés, entreprises). La deuxième s'intéresse à l'adoption de l'administration électronique par les communes. Comme le souligne cette section, les résultats empiriques de ces deux approches s'accordent quant à l'influence des caractéristiques socioéconomiques sur les comportements d'adoption.

\subsection{Les déterminants liés à l'usage des services Internet par les administrés}

De nombreuses études empiriques analysent l'adoption et l'usage des services Internet du point de vue des ménages. Les premiers travaux français, largement inspirés par les travaux menés aux États-Unis, ont porté sur les déterminants d'adoption des TIC (Le Guel et al. 2004, 2005). Menés sur des données luxembourgeoises (2004) puis bretonnes (2005), ces travaux

\footnotetext{
8 Il s'agit ici de distinguer les services caractérisés par une obligation légale de dématérialisation (dématérialisation imposée par le programme gouvernementale pour l'Administration ELEctronique (Adèle) par exemple) des services qui émanent des initiatives propres des communes.
} 
ont par exemple montré une influence significative des caractéristiques socioéconomiques des ménages (âge, niveau d'études, caractéristiques socioprofessionnelles, etc.) sur le comportement d'adoption et d'usage des TIC. Le Guel et al. (2004, 2005) soulignent par exemple qu'un âge inférieur à 64 ans et la catégorie socioprofessionnelle du chef de famille (cadres et professions intermédiaires) jouent positivement sur la probabilité d'adopter une connexion Internet à domicile. Bayo-Moriones et Lera-Lopez (2007) ont également validé l'hypothèse selon laquelle le niveau d'éducation (niveau d'études et catégorie socioprofessionnelle) est positivement et significativement corrélé à la probabilité d'adopter les TIC. Ils nuancent en revanche l'influence de l'âge des salariés.

La méthodologie adoptée par ces travaux s'est ensuite généralisée à l'analyse des déterminants d'adoption des services de commerce électronique (Le Guel et al., ibid ; Suire, 2007) et des services d'administration électronique centrale. La population d'usagers étudiée est soit les citoyens (Suire, 2007 ; Bacache-Beauvallet et al., 2011) soit les entreprises (Martin et Poussing, 2007) ${ }^{9}$. Suire (2007) compare par exemple les caractéristiques socioéconomiques des usagers des services de commerce électronique et les caractéristiques socioéconomiques des usagers des services d'administration électronique centrale. Il met en évidence deux comportements : les usagers «citoyens» et les usagers «citadins». Les premiers ont une intensité d'usage des services de commerce électronique plus forte que les deuxièmes. Inversement, les usagers « citadins » sont caractérisés par une intensité d'usage plus forte des services d'administration électronique que les usagers « citoyens ». Les usagers «citadins » ont une vie sociale riche et ont tendance à se détacher des choses de la vie publique, alors que les usagers «citoyens » sont plus intéressés par la vie de la cité et s'engagent davantage dans

\footnotetext{
${ }^{9}$ Une deuxième littérature s'est récemment développée (Susanto et Goodwin, 2010). Elle analyse l'adoption des services d'administration électronique à l'appui des modèles théoriques d'adoption des innovations répandus, en particulier le modèle d'acception de la technologie (TAM) de Davis (1989). Cette littérature est difficilement mobilisable dans le cadre de notre recherche dans la mesure où l'objet analysé sont les facteurs à l'origine des intentions d'adoption ou de l'acceptation d'un service appréhendés à travers les attributs du service.
} 
la vie associative. Leurs usages d'Internet viennent ainsi, selon Suire (2007), dans le prolongement de leur trajectoire citoyenne.

Autre exemple, les travaux de Bacache-Beauvallet et al. (2011) analysent, à l'appui d'une enquête menée en 2005 par l'INSEE auprès d'un échantillon de 5603 français, l'usage des services d'administration électronique fiscale (c'est-à-dire les services proposés sur le site impot.gouv.fr), les services liés au marché du travail (site de l'ANPE et de l'Assedic), les services proposés par le site de la Caisse nationale d'assurance maladie. Les auteurs observent que les actifs les plus éduqués sont ceux qui utilisent le plus les sites administratifs. Ils concluent que le développement des services est fortement lié aux compétences des particuliers en matière de nouvelles technologies.

\subsection{Les déterminants internes aux communes}

Du point de vue des communes, l'attention a dans un premier temps été portée sur la mise en place ou non d'un site Internet communal. Puis la littérature a dans un deuxième temps cherché à identifier les déterminants à l'origine de ce choix. Un des premiers déterminants traditionnellement mis en évidence est la personnalité du maire plus ou moins ouvert aux TIC comme cela a été le cas par exemple pour la ville de Parthenay (Vidal, 2007). La taille de la population est un deuxième déterminant communément reconnu comme significativement influant sur l'implication des communes dans la numérisation des services d'administration (Brudney et Selden, 1995 ; Coursey et Norris 2008 ; Moon, 2000 ; Moon et Norris, 2005 ; Weare et al. 1999, Dang Nguyen et al., 2011). Pour Dang Nguyen et al. (2011), avec la taille de la population, l'environnement touristique des communes est un autre déterminant qui influence positivement et significativement le niveau de développement de l'administration électronique dans le cas des communes bretonnes. Enfin, plus généralement, plusieurs travaux empiriques confirment le rôle fondamental des variables socioéconomiques et 
démographiques dans les choix des acteurs publics locaux en matière de développement de l'administration électronique (Finney et Yoon, 2011 ; Jun et Weare, 2008 ; Lee et Berry, 2011).

Ces travaux identifient les facteurs qui influencent le développement de l'administration électronique locale. Une dichotomie est en fait généralement établie entre infrastructures et services dans les travaux sur les TIC. La présente recherche vise à dépasser cette dichotomie. Il s'agit d'identifier comment les communes orientent leurs investissements en matière de déploiement des services Internet, i.e. sont-elles :

- uniquement orientées 'développement de l'administration électronique (dimension services)' ?

- uniquement 'orientées déploiement des technologies d'accès aux TIC (dimension technologies d'accès)' ?

- à la fois orientées 'déploiement des technologies d'accès et développement de l'administration électronique (interdépendances entre technologies d'accès et services)' ?

Un deuxième enjeu sera d'identifier les déterminants qui orientent les choix des communes. Il s'agit ici de considérer les enseignements de la littérature résumés ci-dessous : 


\begin{tabular}{|c|c|}
\hline \multicolumn{2}{|c|}{$\begin{array}{l}\text { Enseignements de la littérature sur le comportement des communes en matière de } \\
\text { déploiement des infrastructures et des services d'administration électronique }\end{array}$} \\
\hline $\begin{array}{l}\text { Les communes amenées à investir dans le déploiement } \\
\text { des technologies d'accès aux TIC sont les communes à } \\
\text { faible densité de population, rurales ou localisées en } \\
\text { régions périphériques (dans une logique de rattrapage). }\end{array}$ & $\begin{array}{l}\text { Vicente et Suire (2004), Nguyen et } \\
\text { Vicente (2001), Vicente (2005), } \\
\text { Rallet et Ullmann (2005) }\end{array}$ \\
\hline $\begin{array}{l}\text { Les caractéristiques socioéconomiques des usagers } \\
\text { influencent leur adoption des équipements en TIC et des } \\
\text { services Internet (de commerce électronique comme } \\
\text { d'administration électronique) }\end{array}$ & $\begin{array}{l}\text { (2011), } \\
\text { Lopez }(2007)\end{array}$ \\
\hline $\begin{array}{l}\text { La taille et la densité de la population influencent } \\
\text { l'adoption de l'administration électronique par la } \\
\text { commune. } \\
\text { L'environnement touristique et les caractéristiques } \\
\text { socioéconomiques d'une commune influencent } \\
\text { l'adoption de l'administration électronique par la } \\
\text { commune }\end{array}$ & $\begin{array}{l}\text { Brudney et Selden, (1995); Coursey et } \\
\text { Norris (2006); Moon, (2000); Moon } \\
\text { et Norris, (2005); Galliano et Roux } \\
(2006) \text {; Weare et al. (1999); Dang } \\
\text { Nguyen } \text { et al. (2011); Finney et Yoon, } \\
\text { (2011); Jun et Christopher Weare, } \\
\text { (2008); Lee et Berry, (2011) }\end{array}$ \\
\hline
\end{tabular}

\section{Méthodologie}

Cette recherche est essentiellement empirique : elle s'attache d'abord à construire une base de connaissance originale sur le développement de l'administration électronique et le niveau de déploiement des technologies d'accès sur le territoire des communes françaises, puis à en analyser les fondements socioéconomiques. L'objectif est de construire un échantillon représentatif pertinent au regard du problème posé et de produire l'information empirique nécessaire. Cela a nécessité l'élaboration d'un questionnaire qui synthétise les développements et puisse répondre aux questionnements mis en évidence dans la littérature. Cette partie explicite ces différents éléments et présente une synthèse rapide des résultats relatifs à l'état de dématérialisation des services d'administration électronique locale. 


\subsection{Le choix de l'échantillon}

La construction de la base de données a bénéficié du soutien d'Orange Labs Sophia-Antipolis dans le cadre d'une convention CIFRE au cours de laquelle un projet (COLLUP: «COLlectivités Locales Usages et Processus ») a été mené. Ce soutien a permis la réalisation d'une enquête, conduite en 2007, auprès d'un échantillon représentatif de communes. Elle a interrogé les directeurs du service informatique (DSI) et les chargés de mission TIC des communes sur la nature et le niveau de déploiement du haut et très haut débit d'une part, sur l'état de la dématérialisation des services d'administration locale d'autre part.

Les communes de l'échantillon ont été sélectionnées en croisant deux premiers critères : une représentativité géographique en cinq zones (Ile de France, Grand Ouest, Nord Est, Sud Est, Sud-Ouest) et un critère de taille. On le sait, la France compte plus de 36000 communes, dont la population varie de quelques dizaines à quelques millions d'habitants, avec une médiane de 420 habitants. Notre échantillon ne s'attache pas à représenter cette réalité. Il est construit selon un critère de taille, mais au regard de la pertinence avec la question de recherche posée. Cela a conduit à fixer des bornes à la taille des communes considérées.

Une borne inférieure : 10000 habitants. En effet, 84\% de l'ensemble des communes françaises avaient un site Internet en 2007, condition nécessaire au développement de l'administration électronique. Mais cette proportion s'élevait à $99 \%$ pour les villes de plus de 10000 habitants et tombait à moins de $6 \%$ pour celles de moins de 10000 habitants (Fourgous, 2010).

Une borne supérieure : 100000 habitants. Les communes de plus de 100000 habitants sont définies par l'ARCEP comme les 'zones très denses'. Il s'agit en réalité des quelques grandes zones urbaines où se concentrent les activités et où les opérateurs se livrent une concurrence sévère dans le haut ou le très haut débit (ACUF, 2010). Plus précisément, ce sont des régions où une concentration de type «réseaux métropolitains» a été mise en évidence par la 
littérature (Dang Nguyen et Vicente, 2005) qui défend l'hypothèse suivante : lorsqu'elles investissent dans les infrastructures numériques, les problématiques mises en place par ces communes sont très spécifiques : elles ne renvoient pas aux prérogatives de la LCEN.

On a vu en effet que depuis 2004 la LCEN autorise les collectivités à déployer, exploiter des réseaux de communications électroniques et à fournir des services de télécommunication. Dans la mesure où les projets de collectivités consistent à subventionner un opérateur dans le cadre d'une Délégation de Service Public $\left(\mathrm{DSP}^{10}\right)$ ou d'un Partenariat Public-Privé $\left(\mathrm{PPP}^{11}\right)$ par exemple, ils sont soumis à la réglementation communautaire sur les aides d'Etat qui n'autorise à subventionner des opérateurs que dans certains cas et avec certaines contreparties. En particulier, la LCEN prévoit que dans les zones les plus rentables (les 'zones très denses', où plusieurs opérateurs sont susceptibles de déployer leurs réseaux), une intervention publique sous forme de subvention n'est pas conforme au droit européen car elle risque de perturber la concurrence entre les opérateurs. En revanche, dans les zones moins denses, où les opérateurs ne prévoient pas de déployer, cette intervention a toute sa légitimité (ACUF, 2010 ; Marty, 2013).

La classe [10 000 - 100000$]$ habitants que nous avons choisie est la plus pertinente pour notre propos parce que ces communes sont au cœur des enjeux de développement numérique, de la relation technologies d'accès - services à mettre en place. Cette classe de population représente un total de 815 communes en France.

L'échantillon considéré dans ce travail a été construit à partir du panel représentatif de cette classe de communes mis en place par Orange Labs dans le cadre du projet Collup. Cet échantillon compte 287 communes interrogées par questionnaire envoyé en ligne ou

\footnotetext{
${ }^{10}$ Les délégations de service public désigne l'ensemble des contrats par lesquels une personne morale de droit public confie la gestion d'un service public dont elle a la responsabilité à un délégataire public ou privé dont la rémunération est substantiellement liée au résultat d'exploitation du service.

${ }^{11}$ Le partenariat public-privé (PPP) est un mode de financement par lequel une autorité publique fait appel à des prestataires privés pour financer et gérer un équipement assurant ou contribuant au service public. Le partenaire privé reçoit en contrepartie un paiement du partenaire public et/ou des usagers du service qu'il gère.
} 
sollicitées par téléphone dans le cadre d'une enquête originale sur les TIC dans les communes françaises. Cependant seules 233 réponses effectivement exploitables (au sens de questionnaires correctement remplis, mais aussi de communes disposant du haut débit et d'un site Internet) ont été considérées.

\subsection{Présentation de la base de données}

La base de données est constituée de deux volets renseignés pour chacune des communes de l'échantillon.

\section{Le volet TIC de la base de données}

Ce volet est le résultat d'une enquête réalisée en écho aux différentes sources analytiques et empiriques existantes. Il comporte deux parties.

La première partie de l'enquête porte sur le type de technologie déployé dans les communes : technologie haut débit $\left(\mathrm{ADSL}^{12}\right)$, technologie très haut débit (fibre optique ${ }^{13}$ et / ou ADSL2 $+{ }^{14}$ ), autres technologies (wifi, wimax, technologies satellites). Par ailleurs, les communes ont été interrogées sur la nature de l'offre pour la technologie dont le débit est le plus élevé. La réponse à cette question est orientée de manière à identifier la nature de l'effort des acteurs publics locaux, comme des opérateurs de télécommunication, dans la technologie de plus haut débit implantée sur le territoire (Quel acteur est à l'initiative du déploiement de la

\footnotetext{
${ }^{12}$ La technologie Asymmetric Digital Subscriber Line (ADSL) signifie « liaison numérique asymétrique » et désigne une technique de communication numérique qui permet d'utiliser une ligne téléphonique, une ligne spécialisée pour transmettre et recevoir des données numériques de manière indépendante du service téléphonique conventionnel. (cf. Journal Officiel du 27 décembre 2009 ou http://www.legifrance.gouv.fr/affichTexte.do?cidTexte=JORFTEXT000021530617).

${ }^{13}$ La fibre optique est une technologie dite de très haut débit. C'est une technologie de connexion qui permet de transmettre des données à une vitesse très élevée sur de grandes distances.

${ }^{14}$ L'ADSL 2+ est une évolution de l'ADSL qui utilise 511 sous-porteuses au lieu de 255 , grâce à une extension de la bande de fréquences utilisées jusqu'à 2,2 MHz environ
} 
technologie du plus haut débit? un opérateur de télécommunication ou la collectivité ellemême ?).

L'analyse de la base de données donne les résultats suivants : par construction, toutes les communes de l'échantillon sont couvertes en ADSL (réseau haut débit). Un peu moins de la moitié des communes de notre échantillon dispose également du très haut débit de type Adsl2+ (12.5\%) ou fibre optique (26\%). Du point de vue de la nature de l'offre du plus haut débit, on observe que $24 \%$ des communes de l'échantillon ont piloté et ont financé seules le déploiement de la technologie, $31 \%$ des communes ont sollicité un ou plusieurs opérateurs télécommunication et $45 \%$ ont bénéficié d'une offre spontanée de la part d'un opérateur de télécommunications.

La seconde partie apporte une information sur l'état de dématérialisation des procédures administratives qui émanent directement des mairies sollicitées dans le cadre de l'enquête. Les mairies ont été interrogées sur une liste de vingt services d'administration électronique locale à l'instar des travaux d'Attour-Oueslati et al. (2007), Dang Nguyen et Trémenbert (2008), ou de Dang Nguyen et al. (2011). Cet état de dématérialisation est renseigné par différents stades, un stade 0 (le service n'apparaît pas sur le site), un stade 1 (une simple information existe), un stade 2 (téléchargement possible des documents nécessaires à la procédure), un stade 3 (téléchargement des documents et saisie de données sur le formulaire), et un stade 4 (transaction complète).

La liste des services potentiels inclus dans le questionnaire est la suivante : procédure d'appel d'offres de marchés publics, les services liés aux activités touristiques et événements culturels, les services liés aux bibliothèques/médiathèques, les services d'inscriptions en ligne à un service municipal, les services petites enfance, les services de billetteries municipales, les services de consultation citoyen, les offre d'emplois de la mairie, la délivrance de permis de construire, les services d'alertes en cas de catastrophe, les services d'élections, les services 
sociaux, les consultations d'éléments d'urbanismes, les autorisations pour les concessionnaires réseaux, les services liés à l'agenda, les factures fournisseurs, la carte de vie quotidienne, les services FAST $^{15}$. Hormis les procédures d'appel d'offres de marchés publics, pour lesquelles les communes ont une obligation légale, les communes étaient libres de proposer ou pas les différents services, comme de choisir le stade de dématérialisation.

La Figure 1 suivante rapporte cette liste de services et leur stade de dématérialisation tels qu'ils apparaissent pour les communes de notre échantillon. Ces services sont présentés dans la Figure 1 suivante du plus aux moins dématérialisés.

Figure.1 Description de l'état de dématérialisation des services

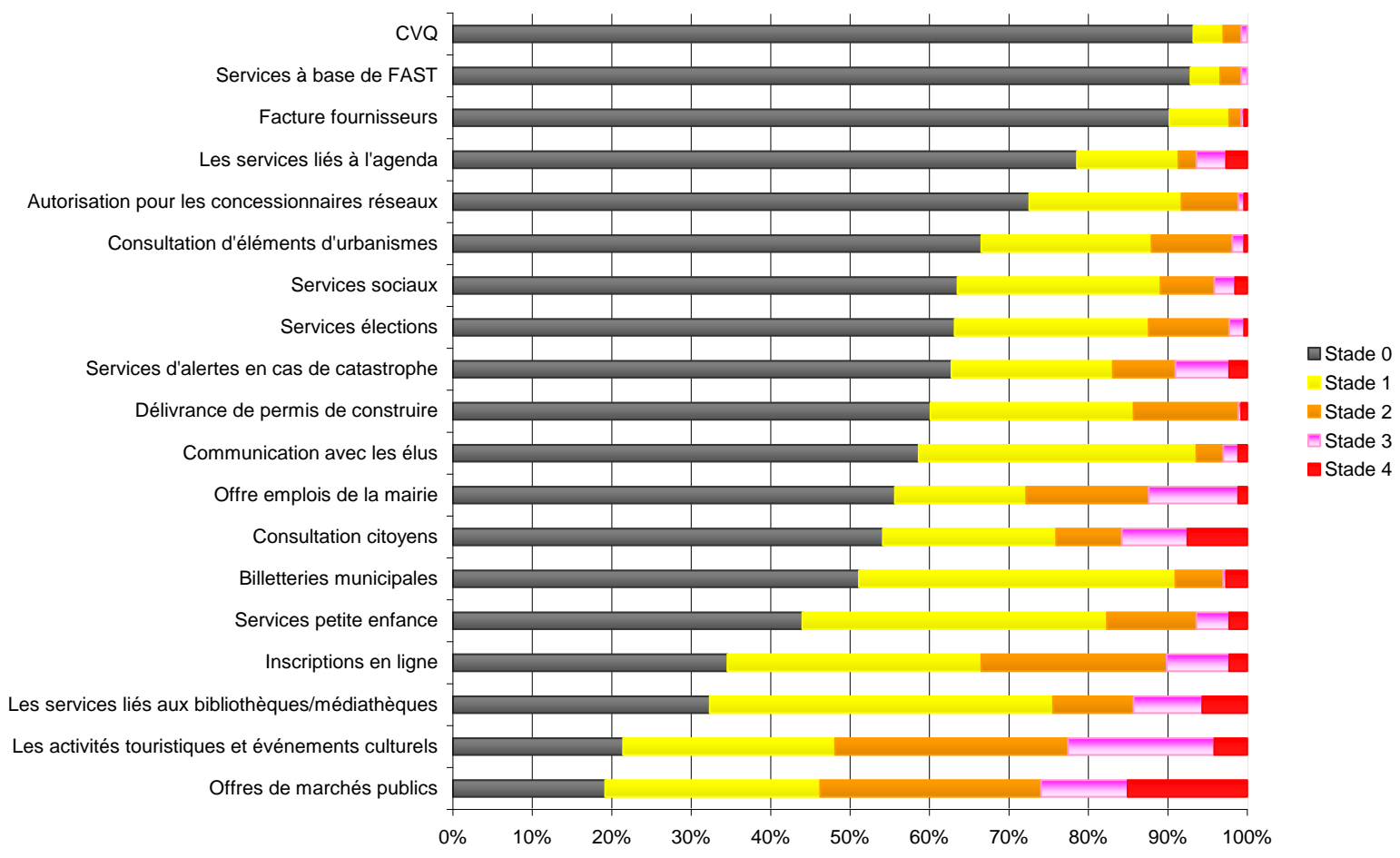

\footnotetext{
${ }^{15}$ Le service « Fournisseur d'accès sécurisé transactionnel » est un outil informatique qui met les technologies de signature électronique au service de la dématérialisation des échanges de tout type de documents présentant une valeur juridique ou financière élevée.
} 
Cet état descriptif montre que dans notre échantillon de communes de plus de 10000 habitants la dématérialisation des services d'administration locale était en phase de décollage en $2007^{16}$. Les résultats descriptifs mettent également en évidence une diversité importante.

\section{Le volet socioéconomique de la base de données}

Le second volet de la base est constitué de données socioéconomiques sur les communes. Il a été élaboré à partir des données publiques publiées sur les communes par l'INSEE et de recherches sur les sites Internet des communes. Ces données renseignent les caractéristiques géographiques (population, densité), économiques (nombre et tailles d'entreprises, répartitions sectorielles) et socioculturelles (répartition des catégories socioprofessionnelles, part des diplômés, des actifs, revenus et impôts des ménages) des communes françaises.

\subsection{Caractéristique de l'échantillon et traitement des données}

Notre échantillon est construit sur la base d'un critère de taille, condition nécessaire à sa cohérence dans la perspective de l'analyse des stratégies de développement numérique des communes. Cependant, la taille n'est pas nécessairement déterminante dans cet ensemble de communes pour expliquer l'état de la dématérialisation. La taille est souvent perçue comme la variable explicative essentielle. Cela est vrai lorsqu'on considère l'échantillon exhaustif des communes d'un pays ou d'une région donnée (Dang Nguyen et al., 2011, Dejean et Souquet, 2011). Mais dans le cadre d'un échantillon plus homogène tel que celui considéré dans cette recherche, la stratégie des communes n'est peut-être pas dictée par des déterminants 'physiques' mais aussi par des déterminants socio-économiques. Le traitement des bases de données entrepris dans la dernière partie de ce travail vise à considérer cette hypothèse, i.e. mettre en évidence une différenciation des communes dans la dématérialisation des services et

\footnotetext{
${ }^{16}$ Sachant qu'il restait environ 36000 communes (sur les 36568 recensées en France métropolitaine) où les conditions préalables à l'e-administration n'étaient pas remplies.
} 
vérifier si elle reflète des différences socioéconomiques. Une classification des communes selon l'état de la dématérialisation des services d'administration électronique locale est d'abord réalisée, puis illustrée par le traitement des bases de données géographiques et socioéconomiques.

\section{Résultats}

L'analyse croisée des volets 'TIC' et 'socioéconomique' de la base de données réalisée dans cette dernière partie vise à mettre en évidence les différentes stratégies de développement de l'administration électronique locale par les communes, qui sont ensuite caractérisée à l'aide des déterminants socioéconomiques des classes de communes.

\subsection{Etat de la dématérialisation et détermination des classes de communes}

L'analyse de la différenciation des communes à partir du niveau de mise en ligne des services d'administration électronique a été effectuée en deux étapes : une analyse de correspondance multiple suivie d'une classification hiérarchique ascendante ${ }^{17}$. L'analyse est réalisée sur les variables qualitatives présentées dans le Tableau 2 qui correspond aux services d'administration électronique locale suffisamment renseignés et effectivement déployés dans les communes considérées au moment de l'enquête.

\footnotetext{
${ }^{17}$ Les résultats de ces analyses sont très sensibles à la distribution des modalités des variables qualitatives considérées; en particulier la part d'inertie due à une modalité est d'autant plus grande que son occurrence est faible, ce qui crée des distorsions artificielles dans la constitution des classes. Les résultats bruts de l'enquête décrits dans le paragraphe précédent, où certaines modalités (les stades 4 et 5 de la dématérialisation) apparaissent peu, et où les stades 0 et 1 sont surreprésentés, ont conduit à recomposer la base de données. Les modalités ont ainsi classiquement été regroupées de 5 à 3 stades (en fusionnant en $1:$ Absence, 2 : Information, 3 : téléchargement de l'information, saisie et transaction).
} 
Tableau 2. Liste des services d'administration électronique locale retenus dans l'analyse

\begin{tabular}{|l|l|}
\hline Variables qualitatives & Libellés \\
\hline $\begin{array}{l}\text { Les inscriptions auprès de services municipaux : crèches, } \\
\text { écoles, cantine, bibliothèque, autres... }\end{array}$ & Services inscriptions (A5S1) \\
\hline Les offres des marchés publics & Marchés publics (A5S2) \\
\hline La délivrance des permis de construire & Permis de construire (A5S3) \\
\hline La consultation d'éléments d'urbanisme (POS, cadastre...) & Urbanisme (A5S4) \\
\hline $\begin{array}{l}\text { Les autorisations pour les concessionnaires des réseaux de } \\
\text { la voie publique (intervention travaux voie publique) }\end{array}$ & $\begin{array}{l}\text { Autorisations pour les concessionnaires } \\
\text { réseaux(A5S5) }\end{array}$ \\
\hline $\begin{array}{l}\text { La consultation des citoyens (les citoyens donnent leur avis } \\
\text { sur des projets municipaux....) }\end{array}$ & Consultation des citoyens (A5S6) \\
\hline $\begin{array}{l}\text { Les services sociaux ou de santé (les citoyens peuvent } \\
\text { accéder à leur dossier) }\end{array}$ & Services sociaux (A5S7) \\
\hline $\begin{array}{l}\text { Les services liés à la petite enfance (assistante maternelle, } \\
\text { baby-sitter, garderie, centre loisirs ...) }\end{array}$ & Services petite enfance (A5S8) \\
\hline $\begin{array}{l}\text { Les billetteries municipales (concerts, musées, } \\
\text { spectacles...) }\end{array}$ & Billetterie municipale(A8) \\
\hline $\begin{array}{l}\text { La publication et la consultation d'offres d'emplois } \\
\text { municipaux }\end{array}$ & Offres emplois (A11) \\
\hline Les activités touristiques ou évènements culturels & Activités touristiques (A29) \\
\hline Les services liés aux bibliothèques / médiathèques & Bibliothèques/médiathèques (A32) \\
\hline
\end{tabular}

Chacune de ces variables a trois modalités caractérisant l'état de la dématérialisation: inexistant (un), décollage (deux), avancé (trois). Une Analyse des Correspondances Multiples (ACM) a été réalisée sur ces variables qualitatives. La Figure 2 ci-dessous synthétise les résultats importants de l'analyse.

Figure.2 Représentation des modalités

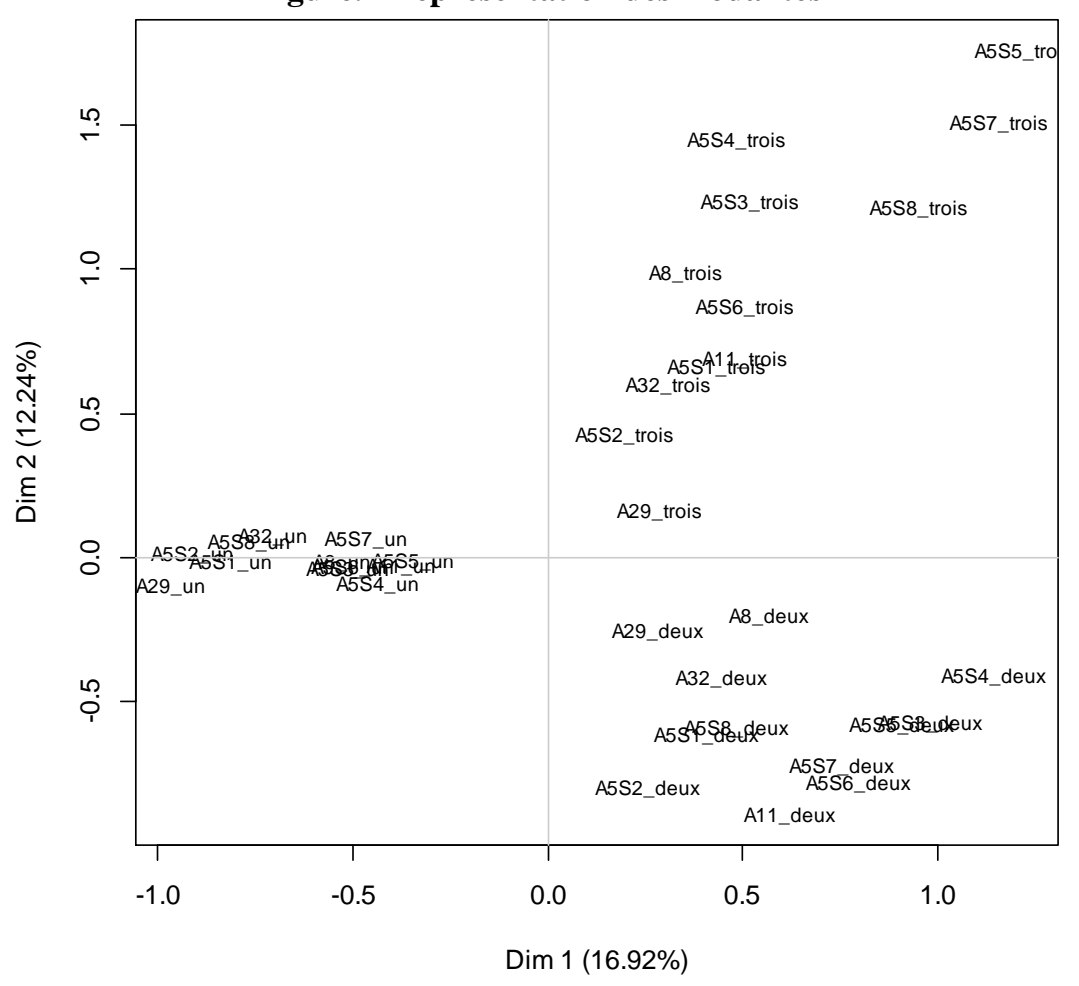


Les deux premiers axes représentent $29.16 \%$ de l'inertie totale, ce qui est relativement important $^{18}$. La Figure 2 est la représentation graphique des modalités des variables qualitatives. Le premier axe oppose les communes qui ne sont pas engagées dans le processus de dématérialisation des services aux autres communes. Le second axe oppose le degré d'engagement dans le processus pour celles qui investissent dans le développement de l'administration électronique. Le premier axe est le plus approprié pour observer les modalités 1, le second pour observer les modalités 2 et 3 .

La classification hiérarchique réalisée sur les coordonnées factorielles de l'ACM permet logiquement d'identifier une partition en trois classes. Comme l'illustre la Figure 2, les modalités des variables diffèrent significativement entre les classes. La liste des variables qui séparent le mieux les communes, ordonnées selon la valeur du $\mathrm{Chi}^{2}$, est donnée par le Tableau 3.

Tableau 3. Les variables explicatives : analyse statistique

\begin{tabular}{|c|c|c|}
\hline Variables & p.value & df \\
\hline Services petite enfance (A5S8) & $5.850468 \mathrm{e}-35$ & 4 \\
Services sociaux (A5S7) & $3.580338 \mathrm{e}-32$ & 4 \\
Permis de construire (A5S3) & $4.234516 \mathrm{e}-23$ & 4 \\
Autorisations réseaux(A5S5) & $2.169194 \mathrm{e}-21$ & 4 \\
Urbanisme (A5S4) & $2.426703 \mathrm{e}-19$ & 4 \\
Consultation des citoyens (A5S6) & $3.793475 \mathrm{e}-14$ & 4 \\
Services inscriptions (A5S1) & $9.499419 \mathrm{e}-14$ & 4 \\
Billetterie municipale(A8) & $1.251232 \mathrm{e}-12$ & 4 \\
Bibliothèques/médiathèques (A32) & $1.410486 \mathrm{e}-12$ & 4 \\
Marchés publics (A5S2) & $3.431876 \mathrm{e}-08$ & 4 \\
Activités touristiques (A29) & $1.039154 \mathrm{e}-07$ & 4 \\
Offres emplois (A11) & $4.561216 \mathrm{e}-07$ & 4 \\
\hline
\end{tabular}

Les variables qui séparent le mieux les communes sont ainsi les Services petite enfance, puis les Services sociaux et les Services permis de construire.

\footnotetext{
${ }^{18}$ Dans une ACM le nombre de facteurs est égal au nombre de modalités moins le nombre de variables, soit ici 24. Les deux premiers facteurs résument $29.16 \%$ de l'information contenue dans le tableau, le poids des suivants décroit rapidement.
} 
La Figure 3 synthétise les résultats de la classification hiérarchique effectuée sur les coordonnées factorielles de l'ACM. Les classes (Classe 1, Classe 2, Classe 3 dans la suite) comptent respectivement 117, 87, et 19 communes. Elles se caractérisent par une implication croissante dans le déploiement des services d'e-administration, la Classe 3, limitée à 19 communes, étant la classe des communes les plus avancées dans la dématérialisation des services.

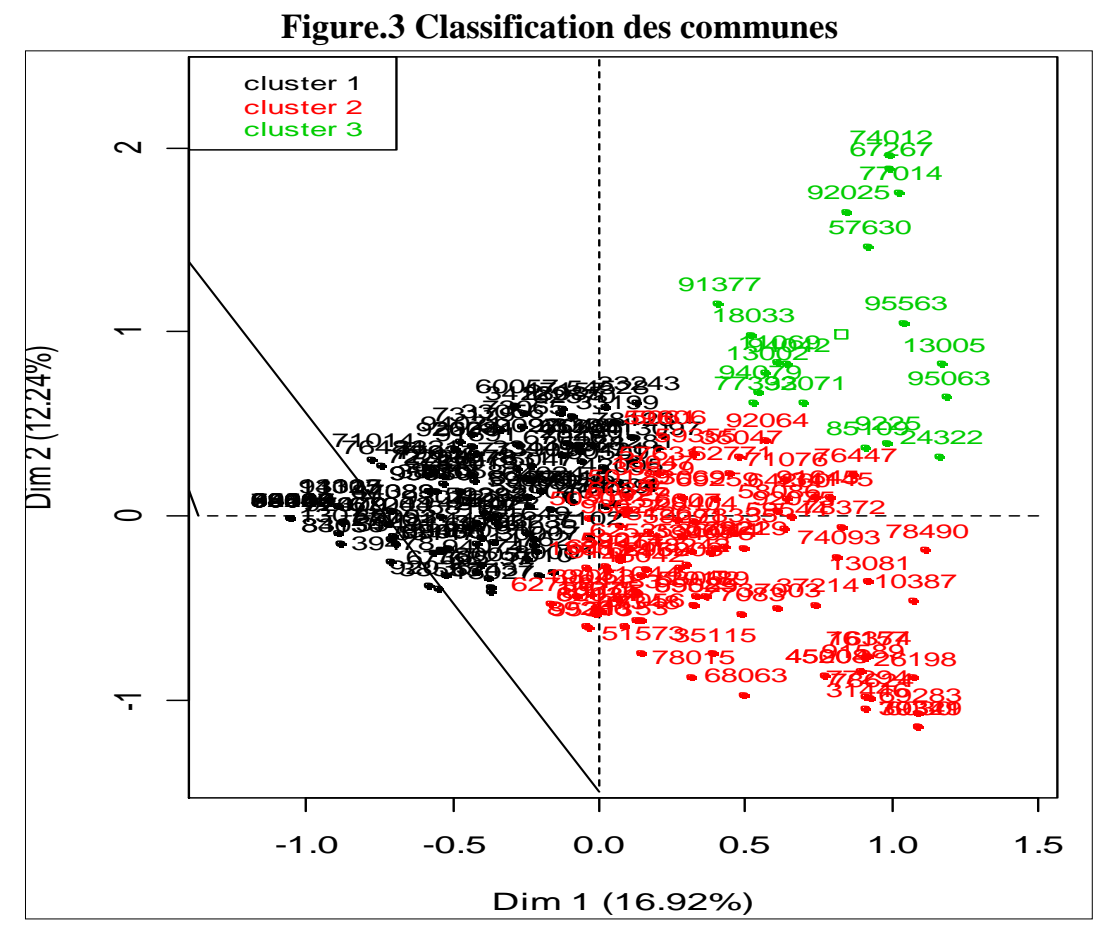

Le Tableau 4 permet enfin de caractériser précisément chaque classe selon les variables et les modalités. Il donne la part des classes par modalité, la part des modalités par classes, le poids global de la modalité considérée et la significativité des résultats. Ce tableau présente les résultats pour les trois classes, en se limitant aux quatre modalités les plus significatives pour chacune des classes 
Tableau 4. Caractéristiques des classes de communes

\begin{tabular}{|c|c|c|c|c|c|c|}
\hline \multicolumn{2}{|c|}{ Classe 3} & \multirow{2}{*}{$\begin{array}{c}\text { Cla/Mod } \\
45.9459459\end{array}$} & \multirow{2}{*}{$\begin{array}{c}\text { Mod/Cla } \\
89.473684\end{array}$} & \multirow{2}{*}{\begin{tabular}{|c|} 
Global \\
16.591928
\end{tabular}} & \multirow{2}{*}{$\begin{array}{c}\text { p.value } \\
3.575848 \mathrm{e}-13\end{array}$} & \multirow{2}{*}{$\begin{array}{c}\text { v.test } \\
7.270717\end{array}$} \\
\hline Services petite enfance : & A5S8_trois & & & & & \\
\hline Services sociaux : & A5S7_trois & 61.9047619 & 68.421053 & 9.417040 & $2.340568 \mathrm{e}-11$ & 6.683027 \\
\hline Autorisations pour les & A5S5_trois & 66.6666667 & 52.631579 & 6.726457 & $6.668498 \mathrm{e}-09$ & 5.799067 \\
\hline concessionnaires réseaux : & & & & & & \\
\hline Permis de construire : & A5S3_trois & 40.0000000 & 63.157895 & 13.452915 & $2.086526 \mathrm{e}-07$ & 5.191459 \\
\hline$\ldots / \ldots$ & $\ldots / \ldots$ & $\ldots / \ldots$ & $\ldots / \ldots$ & $\ldots / \ldots$ & $\ldots / \ldots$ & $\ldots / \ldots$ \\
\hline \multicolumn{2}{|c|}{ Classe 2} & Cla/Mod & Mod/Cla & Global & p.value & v.test \\
\hline Services petite enfance : & A5S8_deux & 75.581395 & 74.712644 & 38.56502 & $3.050594 \mathrm{e}-19$ & 8.966870 \\
\hline Permis de construire : & A5S3_deux & 83.928571 & 54.022989 & 25.11211 & $1.606616 \mathrm{e}-15$ & 7.968462 \\
\hline Services sociaux : & A5S7_deux & 82.456140 & 54.022989 & 25.56054 & $7.061723 \mathrm{e}-15$ & 7.783371 \\
\hline \multirow[t]{2}{*}{ Bibliothèques/médiathèques: } & A32_deux & 65.934066 & 68.965517 & 40.80717 & $7.488423 \mathrm{e}-12$ & 6.848010 \\
\hline & $\ldots / \ldots$ & $\ldots / \ldots$ & $\ldots / \ldots$ & $\ldots / \ldots$ & $\ldots / \ldots$ & $\ldots / \ldots$ \\
\hline \multicolumn{2}{|c|}{ Classe 1} & Cla/Mod & Mod/Cla & Global & p.value & v.test \\
\hline Services petite enfance : & A5S8_un & 88.000000 & 75.213675 & 44.843049 & $5.039944 \mathrm{e}-23$ & 9.880858 \\
\hline Services sociaux & A5S7_un & 74.482759 & 92.307692 & 65.022422 & $3.064902 \mathrm{e}-20$ & 9.216663 \\
\hline Permis de construire : & A5S3_un & 71.532847 & 83.760684 & 61.434978 & $5.818140 \mathrm{e}-13$ & 7.204677 \\
\hline Services inscriptions : & A5S1_un & 84.210526 & 54.700855 & 34.080717 & $4.673425 \mathrm{e}-12$ & 6.915158 \\
\hline & $\ldots / \ldots$ & $\ldots / \ldots$ & $\ldots / \ldots$ & $\ldots / \ldots$ & $\ldots / \ldots$ & $\ldots / \ldots$ \\
\hline
\end{tabular}

Ainsi, $45.945 \%$ des modalités 'avancé' de la variable Services petite enfance appartiennent à la Classe 3 qui ne compte que 19 communes. $89.47 \%$ des communes de la Classe 3 ont la modalité 'avancé' (A5S8_trois) pour la variable Services petite enfance, alors que cette proportion tombe à $16.6 \%$ pour l'ensemble. Ces proportions sont tout aussi marquées pour les Services sociaux et les Services permis de construire, dont les modalités 'avancé' sont à 62 et $66 \%$ respectivement dans la Classe 3. Cette répartition est quasiment opposée pour la Classe 1, qui regroupe les communes en retard. Ainsi, $88 \%$ des communes dont la modalité de la variable Services petite enfance est 'inexistant' appartiennent à la Classe 1 qui compte $52 \%$ des communes de l'échantillon étudié. $75.2 \%$ des communes de cette Classe 1 ont la modalité 'inexistant' pour la variable Services petite enfance, quand cette part est de $44.8 \%$ 
pour l'ensemble. La Classe 2, qui compte 87 communes, est représentative des modalités intermédiaires. $75 \%$ des communes dont la modalité de la variable Services petite enfance est 'deux' appartiennent à la Classe 2. $74 \%$ des communes de cette même Classe 2 ont cette modalité.

\subsection{Les technologies d'accès des classes de commune}

Les différentes classes de communes ont enfin été mises en relation avec différentes informations relatives aux technologies d'accès. Le Tableau 5 suivant croise les 3 classes avec la question interrogeant les communes sur la nature de leur effort quant au réseau de plus haut débit déployé sur leur territoire. Les résultats ne montrent pas de différences significatives dans l'implication des communes. Les mêmes parts relatives des différentes stratégies de déploiement parcourent en effet les différentes classes. En outre, les résultats sont représentatifs de communes non métropolitaines, les opérateurs ne déploient pas spontanément le réseau compte tenu de la taille des marchés.

Tableau 5. Rôle des Collectivités dans le déploiement du réseau de plus haut débit

\begin{tabular}{l|l|l|l|l|}
\hline Classes & Déploiement 1 & Déploiement 2 & Déploiement 3 \\
\hline 1 & 26 & 42 & 49 \\
\hline 2 & 23 & 24 & 40 \\
\hline 3 & 5 & 6 & 8 \\
\hline
\end{tabular}

Les classes de communes sont enfin caractérisées par les technologies de haut débit avancées disponibles : 
Tableau 6. Technologies haut débit présentes dans les communes

\begin{tabular}{|c|c|c|c|c|c|c|}
\hline \multirow[t]{2}{*}{ Classes } & \multicolumn{2}{|c|}{ ADSL+ (\%) } & \multicolumn{2}{|c|}{ CPL (\%) } & \multicolumn{2}{|c|}{ Fibre (\%) } \\
\hline & oui & non & oui & non & oui & non \\
\hline 1 & 14.5 & 85.5 & 2.6 & 97.4 & 28.2 & 71.8 \\
\hline 2 & 9.2 & 90.8 & 1.1 & 98.9 & 23. & 77.0 \\
\hline 3 & 5.3 & 94.7 & 0.0 & 100.0 & 10.5 & 89.5 \\
\hline
\end{tabular}

Le Tableau 6 montre que les communes de la Classe 3 sont très en retrait par rapport aux autres tant pour le déploiement des technologies ADSL+ que pour les technologies Courants Porteurs en Ligne et la Fibre Optique. Apparemment les autres communes ont plus fait le choix de technologies avancées (haut et très haut débit) que du déploiement de services locaux, probablement sous la pression d'entreprises et de besoin de capacités importante pour la production industrielle. Mais plus simplement, les communes sont probablement restées ancrées sur un problème de fracture numérique primaire, i.e. l'illusion d'un progrès nécessairement entraîné par le seul déploiement de technologies avancées, bien souvent en fait la construction de 'cathédrales dans le désert' (Morgan, 1997). On retrouverait avec les technologies Internet les travers habituels des politiques de développement. Les infrastructures sociales nécessaires à la mise en œuvre et l'utilisation des infrastructures physiques sont souvent absentes alors qu'elles sont des conditions nécessaires au développement local. Les stratégies mises en place par les communes de la Classe 3 semblent obéir à cet impératif : le déploiement de services d'administration électronique locale est aussi une incitation au développement des usages par les citoyens et peut avoir des retombées en termes de développement local ou d'attractivité de leur territoire. 


\subsection{Caractérisation du niveau de dématérialisation des services d'administration des communes}

Ce paragraphe s'efforce de mettre en évidence les spécificités des différentes classes de communes dans le processus de dématérialisation de leurs services d'administration locale. Il caractérise les parangons, les individus spécifiques, et les caractéristiques géographiques, économiques et socioculturelles des trois classes de communes.

\section{Parangons}

Les parangons donnent les communes les plus proches du centre de leur classe. Ils sont représentatifs des communes de la classe. Dans le Tableau 7 suivant, on donne les cinq premières communes par classes, décrites par leur Code Commune INSEE et leur Population.

Tableau 7. Les Parangons

\begin{tabular}{|c|c|c|c|c|c|}
\hline \multicolumn{6}{|c|}{ Classe 1} \\
\hline Code Commune & 45284 & 94022 & 38563 & 64024 & 93072 \\
\hline Population & 17758 & 34336 & 19794 & 35263 & 32839 \\
\hline \multicolumn{6}{|c|}{ Classe 2} \\
\hline Code Commune & 62160 & 78242 & 28404 & 95203 & 59291 \\
\hline Population & 44859 & 12582 & 11496 & 22882 & 16029 \\
\hline \multicolumn{6}{|c|}{ Classe 3} \\
\hline Code Commune & 94042 & 13002 & 11069 & 94079 & 92071 \\
\hline Population & 17117 & 18907 & 43950 & 26632 & 19494 \\
\hline
\end{tabular}

On voit que pour les parangons la population n'est pas une variable discriminante parmi les classes, la commune la plus centrale pour la Classe 3 ne compte que 17117 habitants (avec une moyenne générale pour l'échantillon considéré de 22001 et une médiane de 16 763). Comparativement, les communes centrales de la Classe 1, la classe en retard dans le processus de dématérialisation, sont plus peuplées. Ainsi, Joinville le Pont est la commune qui représente le mieux la Classe 3. 


\section{Individus spécifiques}

Les individus spécifiques donnent les communes les plus éloignées des centres des autres classes. Là encore on donne le code Commune et la population des communes spécifiques (Tableau 8).

Tableau 8. Les communes spécifiques

\begin{tabular}{|c|c|c|c|c|c|}
\hline \multicolumn{7}{|c|}{ Classe 1 } \\
\hline Code Commune & 71014 & 44047 & 59360 & 62587 & 62643 \\
\hline Population & 16419 & 16419 & 20869 & 10558 & 32839 \\
\hline \multicolumn{7}{|c|}{ Classe 2 } \\
\hline Code Commune & 30341 & 79329 & 06030 & 69283 & 37003 \\
\hline Population & 10261 & 10656 & 42158 & 10283 & 11457 \\
\hline \multicolumn{7}{|l|}{ Classe 3 } \\
\hline Code Commune & 67267 & 74102 & 92025 & 77014 & 57630 \\
\hline Population & 16860 & 27253 & 76757 & 14030 & 13330 \\
\hline
\end{tabular}

Les communes spécifiques ne se distinguent pas par la taille. Elles sont parmi les plus petites et sont de tailles comparables. La commune spécifique de la Classe 3, la commune la plus éloignée des centres des Classes 1 et 2 , et que l'on peut considérer comme la plus représentative de la Classe 3 est Lingolsheim. Autun et Vauvert représentent respectivement les Classes 1 et 2.

Ces résultats incitent ainsi à rechercher des explications complémentaires à la taille pour expliquer la classification des communes. Ainsi, les variables quantitatives issues du volet socio-économique de la base de données ont permis de construire un ensemble d'indicateurs sur les communes, qui ont été introduits comme variables illustratives dans l'ACM et la classification hiérarchique. Il apparaît que les variables Spécialisation dans les Services, Part des Diplômés et Nombre d'Entreprises ont les rapports de corrélation significatifs les plus 
élevés avec la variable de classe, la variable Population arrive ensuite, avec une p-value de 0.07 .

Tableau 9. Variables illustratives : caractérisation des classes

\begin{tabular}{|l|l|l|}
\hline & v.test & p.value \\
\hline Classe 3 & & \\
\hline Nombre d'Entreprises & 2.332419 & 0.01967864 \\
Densité & 2.218442 & 0.02652474 \\
Population & 2.146820 & 0.03180760 \\
Spécialisation Services Marchands & 2.087065 & 0.03688222 \\
Spécialisation Services & 1.948001 & 0.05141481 \\
Part des Diplômés & 1.876858 & 0.06053763 \\
Part des Catég. Socio-Pro Supérieures & 1.863521 & 0.06238894 \\
\hline Classe 2 & & \\
\hline Gini36 & 1.881224 & 0.05994145 \\
\hline Classe 1 & & \\
\hline Part des Diplômés & -2.005158 & 0.04494613 \\
Spécialisation Services & -2.031556 & 0.04219865 \\
Niveau Moyen Impôts Ménages Imposés & -2.080569 & 0.03747336 \\
\hline
\end{tabular}

Les variables quantitatives illustratives permettent ensuite de caractériser les différentes classes (Tableau 9). Les communes de la Classe 1 ont ainsi une Part des Diplômes, une Spécialisation dans les Services et un Niveau Moyen des Impôts de Ménages Imposés inférieures à la moyenne (v-test négatifs et significatifs). Les communes de la Classe 3 sont elles caractérisées par un Nombre d'Entreprises, une Densité, une Population, une part des Services et des Services Marchands supérieurs à la moyenne (v-test positifs et significatifs). Les communes de la Classe 2, intermédiaires, ne se distinguent de façon significative que par le Gini36.

Les derniers paragraphes illustrent enfin les communes des différentes classes selon ces caractéristiques socio-économiques ${ }^{19}$. L'influence des classes sur les variables socioéconomiques a été analysée par des analyses de la variance ou des tests de kruskal-wallis. Les effets significatifs dans les modèles correspondent bien sûr essentiellement aux variables illustratives caractéristiques des classes. Il est cependant intéressant dans le cas d'effet significatif d'effectuer des tests de comparaison par paires (respectivement tests de Tukey HSD ou tests de rang de wilcoxon selon le modèle testé), pour identifier quelles sont les

\footnotetext{
${ }^{19}$ Les différentes variables quantitatives satisfont le test de Levene pour le facteur Classe.
} 
paires de classes où ces effets sont significatifs (on retient jusqu'à des p-values de 0.1 ). On ne présente pas l'ensemble de ces tableaux dans la suite, essentiellement descriptive, mais on s'y réfère dans les commentaires.

\section{Les variables géographiques}

Le tableau 10 suivant présente les moyennes et médianes des variables Population, Densité et Taux de Croissance entre 1990 et 1999 des communes.

Tableau 10 Les variables géographiques

\begin{tabular}{|l|l|c|c|c|}
\hline \multicolumn{2}{|c|}{ Classes } & 1 & 2 & 3 \\
\hline Population & Moy. & 20794 & 22235 & 28367 \\
& Med. & 16763 & 16100 & 19494 \\
& & 1951 & 2020 & 3292 \\
\hline Densité & Moy. & 1184 & 1084 & 2963 \\
\cline { 2 - 2 } & Med. & 0.035 & 0.035 & 0.034 \\
\hline Taux de & Moy. & 0.015 & 0.014 & 0.023 \\
\cline { 2 - 2 } croissance & Med. & & & \\
\hline
\end{tabular}

Les moyennes de la taille des Classes 1, 2 et 3 suivent une relation monotone croissante, mais les médianes sont inversées pour les Classes 1 et 2 (Tableau 10). Les .moyennes de la population des communes de Classes 1 et 3 sont significativement différentes, en revanche les communes de la Classe 1 et de la Classe 2 ne peuvent être différenciées. Les taux de croissance moyens sont sensiblement égaux, les effets ne sont pas significatifs. En revanche, la densité est beaucoup plus importante pour la Classe 3. La densité des communes de la Classe 3 est significativement différente de celle des Classes 1 et 2 ; en revanche les communes des Classes 1 et 2 ne peuvent encore être distinguées (p-value $=0.98$ ). Les résultats traduisent l'influence d'un milieu urbain sur le développement numérique. Ce résultat est en phase avec les principaux résultats recensés par la littérature. 


\section{Les variables économiques}

Les données disponibles ont permis de construire différents indicateurs et ratios présentés dans le Tableau 11 suivant.

Tableau 11 Les variables économiques

\begin{tabular}{|c|c|c|c|c|}
\hline \multicolumn{2}{|l|}{ Classes } & 1 & 2 & 3 \\
\hline \multirow[t]{2}{*}{ Gini36 } & Moy. & 0.658 & 0.669 & 0.644 \\
\hline & Med. & 0.659 & 0.669 & 0.648 \\
\hline \multirow{2}{*}{$\begin{array}{l}\text { Nombre moyen de salaries } \\
\text { par entreprise }\end{array}$} & Moy. & 6 & 6 & 5 \\
\hline & Med. & 5 & 5 & 4 \\
\hline \multirow{2}{*}{$\begin{array}{l}\text { Part des entreprises de plus } \\
\text { de } 50 \text { salariés }\end{array}$} & Moy. & 0.017 & 0.017 & 0.014 \\
\hline & Med. & 0.015 & 0.016 & 0.013 \\
\hline \multirow{2}{*}{$\begin{array}{l}\text { Part des salariés dans les } \\
\text { entreprises de plus de } 50\end{array}$} & Moy. & 0.425 & 0.437 & 0.376 \\
\hline & Med. & 0.427 & 0.442 & 0.352 \\
\hline \multirow[t]{2}{*}{ Spécialisation : industrie } & Moy. & 0.180 & 0.174 & 0.145 \\
\hline & Med. & 0.156 & 0.133 & 0.138 \\
\hline \multirow[t]{2}{*}{ Spécialisation : services } & Moy. & 0.538 & 0.560 & 0.597 \\
\hline & Med. & 0.526 & 0.574 & 0.598 \\
\hline \multirow{2}{*}{$\begin{array}{l}\text { Spécialisation : services } \\
\text { marchands }\end{array}$} & Moy. & 0.213 & 0.217 & 0.248 \\
\hline & Med. & 0.207 & 0.207 & 0.245 \\
\hline
\end{tabular}

Les différences vont toutes dans le même sens. Elles caractérisent la singularité de la Classe 3 et permettent aussi de distinguer les Classes 1 et 2 qui sont largement équivalentes en termes de taille.

Le Gini36 mesure l'indice de spécialisation des communes à partir de la répartition des emplois en 36 branches d'activités ; l'effet des classes est significatif. Les effets par paires montrent que les communes de la Classe 3 sont relativement moins spécialisées que celles de la Classe 1, traduisant une urbanisation plus importante au sens de Jacobs (1969), i.e. une 
diversification des activités souvent associée dans la littérature à des capacités de changement, d'innovation plus importantes.

L'indice de spécialisation le plus important est celui de la Classe 2. Mais il est intéressant aussi de considérer la nature de la spécialisation. Par exemple, la spécialisation dans les services, et surtout les services marchands, est plus prononcée dans les communes de la Classe 3 (effets par paires avec les autres classes toujours significatifs) et est à un degré moindre dans les communes de la Classe 2. Ces communes ont des caractéristiques qui correspondent aux tissus urbains contemporains, orientés vers les activités de service. En revanche, les communes de la Classe 1 sont spécialisées dans l'industrie. Les spécialisations distinguent les communes des Classes 1 et 2, mais ces effets ne sont cependant pas statistiquement significatifs.

Des différences importantes se retrouvent dans la nature des entreprises pour la Classe 3. En particulier le Nombre d'Entreprises est beaucoup plus important dans cette classe, les comparaisons par paire avec 1 et 2 sont significatives; en revanche rien ne distingue statistiquement les Classes 1 et 2. Le nombre de salariés moyens par entreprises est plus petit dans la Classe 3. Il en est de même pour la part des entreprises de plus de 50 salariés et la part des salariés employés dans les entreprises de plus de 50 salariés où les différences sont marquées. Ces derniers résultats, qui ne sont cependant pas significatifs, suggèrent donc que le tissu économique de cette classe est moins concentré dans de grandes entreprises.

\section{Les variables socioculturelles}

Le dernier Tableau 12 caractérise les variables socioculturelles. Différents ratios ont été construits à partir des données brutes. La caractéristique essentielle est que tous ces ratios croissent de façon monotone de la Classe 1 vers la Classe 3. 
Le premier ratio donne la part des actifs dans la population. Celui-ci est sensiblement plus important dans les communes de la Classe 3, mais les différences ne sont pas significatives. Ces communes sont surtout marquées par un taux de diplômés et une part des catégories socioprofessionnelles supérieures plus importante. Les différences sont significatives; la Classe 1 a toujours des niveaux moins importants.

Tableau 12 Les variables socioculturelles

\begin{tabular}{|c|c|c|c|c|}
\hline \multicolumn{2}{|l|}{ Classes } & & & \\
\hline \multirow[t]{2}{*}{ Part des actifs } & Moy. & 0.542 & 0.541 & 0.552 \\
\hline & Med. & 0.538 & 0.542 & 0.554 \\
\hline \multirow[t]{2}{*}{ Taux diplômés (> bac) } & Moy. & 0.162 & 0.179 & 0.206 \\
\hline & Med. & 0.145 & 0.154 & 0.191 \\
\hline \multirow{2}{*}{$\begin{array}{l}\text { Part catégories socio } \\
\text { professionnelles sup }\end{array}$} & Moy. & 0.174 & 0.181 & 0.204 \\
\hline & Med. & 0.165 & 0.168 & 0.187 \\
\hline \multirow{2}{*}{$\begin{array}{l}\text { Niveau moyen impôts des } \\
\text { ménages imposés }\end{array}$} & Moy. & 2384 & 2648 & 2852 \\
\hline & Med. & 2268 & 2330 & 2424 \\
\hline \multirow[t]{2}{*}{ Part des ménages imposés } & Moy. & 0.530 & 0.541 & 0.583 \\
\hline & Med. & 0.518 & 0.513 & 0.577 \\
\hline
\end{tabular}

Le niveau moyen des impôts des ménages imposés et le taux des ménages imposés dans la population ont les mêmes caractéristiques. Ces résultats traduisent un tissu social plus favorisé, des citoyens plus ouverts aux usages de l'Internet et donc plus demandeurs de services d'administration électronique locale, comme cela a été mis en évidence dans la littérature (cf. Tableau 1). Les caractéristiques socioculturelles mettent en effet en évidence la singularité de la Classe 3, mais permettent aussi de distinguer les Classes 1 et 2, bien les comparaisons pour la paire 1 et 2 ne soient généralement pas significatives. 


\section{Conclusion}

Cet article a proposé une analyse des stratégies de développement des services d'administration électronique des communes françaises de plus de 10.000 habitants. Il a mis en évidence une importante diversité de l'état de la dématérialisation des services d'administration locale. Les communes, relativement libres des niveaux de services d'administration électronique qu'elles souhaitent développer, ne s'impliquent pas toutes de la même manière. Une analyse statistique simple des bases de données originales qui soustendent cette recherche a permis d'identifier différentes classes de communes et différentes stratégies de développement de l'administration électronique.

Une classe, qui compte peu de communes, est beaucoup plus avancée que les autres en termes de dématérialisation de services d'administration. Elle se distingue nettement en termes de taille et surtout de densité comme de spécialisation dans les services. Un retour sur les données montre que les communes de cette classe sont, en dehors de quelques communes de grandes tailles (Bourge, Carcassonne par exemple), essentiellement des communes situées dans la proche périphérie des grandes aires métropolitaines (Ile de France, Bouches du Rhône, Bas Rhin).

Les deux autres classes, l'une ne s'étant pas encore engagée et l'autre étant dans une phase de décollage dans la dématérialisation des services d'administration électronique locale, sont largement similaires en termes de taille et de situation géographique. Elles se distinguent en revanche par leurs caractéristiques socioéconomiques.

En conclusion, les communes qui ne se sont pas encore engagées dans la dématérialisation sont, quelles que soient leurs tailles, des communes industrielles, moins denses et moins dotées que les autres en termes culturels et socioéconomiques. On retrouve indirectement un résultat mis en évidence dans la littérature (cf. Tableau 1). Les autres communes, au caractère 
tertiaire plus marqué, ont des populations dont les usages des TIC sont les plus importants, ce qui les incite à développer une offre idoine.

\section{BIBLIOGRAPHIE}

Abanumy A., Al-Badi A., Mayhew P., (2005), « e-Government website accessibility: in depth evaluation of Saudi Arabia and Oman », The Electronic Journal of e-Government, Vol.3, Issue 3, p. 99-106, available online at www.ejeg.com

ACUF, (2010), Le déploiement du très haut débit : quels enjeux pour les métropoles françaises? Association des communautés urbaines de France, Paris, septembre.

ACSEL, (2009), Les indicateurs de l'économie numérique, bilan 2008, Secrétariat d'Etat chargé de la prospective et du développement de l'économie numérique, Paris.

ANDERSEN K.J., HeNRIKSEN H.Z., (2005), « e-Government maturity models: extension of the Layne and Lee model », Government Information Quaterly, vol.23, p.236-248.

AtKinson R., CASTRo D., Ezell S.J., (2009), The digital road to recovery: a stimulus plan to create jobs, Boost Productivity and Revitalize America, Washington, DC: The Information Technology and Innovation Foundation.

Attour-Oueslati A., Dufresne D., LONGHi C. (2007), L'administration électronique : vers la définition d'indicateurs d'effort et de performances des collectivités locales dans le déploiement de services, in L'Administration Electronique : Réalités et Perspectives, Lavoisier, p.121-143.

AtTour A., Longhi C., (2009), «Fracture numérique, le chaînon manquant. Les services d'eadministration locale dans les communes françaises», Les Cahiers du Numérique, Vol 5, nº1, 2009, p.119-147.

BaCaChe-Beauvallet M., Bounie D., Franois A. (2011), «Existe-t-il une fracture numérique dans l'usage de l'administration en ligne ?», Revue Economique, 2011/2 Vol.62, p.215-235. 
BAKIS H., 2010, TIC et aménagement numérique des territoires, Conférence Digipolis 'Les TIC : des outils au service du développement territorial, Montbelliard, 26-27 Mai.

BAyO-MARIONES A., LERA-LOPEZ F., (2007), « A firm-level analysis of determinants of ICT adoption in Spain », Technovation, Vol. 27, p. 353-366.

Brousseau M. (2003), "Les trois défis de l'administration électronique », in Administration électronique au service des citoyens, Bruxelles, pp. 247-259.

BRUDNEY J.L., SELDEN S.C., (1995), « The adoption of innovation by smaller local governments: the case of computer technology », The American Review of Public Administration, 25 (1), p.71-86.

CaIrnCross F., (2001), The death of distance, Harvard Business School Press, Cambridge, 2001.

CAP GEMinI, (2005), « Online availability of public services: how is Europe progressing? », 4 March, European Commission Directorate General for Information Society and Media.

Clark D., Gillett S., Lehr W., Sirbu M., Fountain J.E., (2003), «Local government stimulation of broadband: effectiveness, e-Government, and economic development", NCDG, paper 21, http: //scholarwprks.urnass.edu/ncdg/21

COURSEY D., NORRIS D.F., (2008), « Models of e-Government: Are they correct? An empirical assessment», Public Administration Review (PAR), Vol.68, Issue 3, p.523-536.

Crandall R., Litan R., (2008), « The effects of broadband deployment on output and employment: a cross-sectional analysis of U.S », Conference on Telecommunications infrastructure and economic performance, Paris, October $16^{\text {th }}-17^{\text {th }}$.

DAGIRAL E., (2007), « La construction de l'administration électronique au prisme des rapports publics », Terminal, dossier « Administration électronique : où en sommes-nous ? », n99-100, pp. 245255.

Dang NguYen G., ViCenTe J., (2003), Entreprises et hauts débits : le rôle des collectivités locales, Paris : rapport OTV et DIGITIP.

DANG NGUYen G., ViCENTE J., (2005), « Quelques considérations sur l'aménagement numérique des territoires: le rôle des collectivités territoriales dans le déploiement des infrastructures de l'économie numérique », XLIème Colloque de l'ASRDLF, Dijon, 5-7 septembre 2005. 
Dang Nguyen G., Dejean S., Souquet A., (2011), Les internautes moteurs des processus d'adoption et de développement de l'E-gouvernement: une étude sur les communes bretonnes, Document de travail, M@rsouin, novembre.

Dang NguYen G., Tremenbert J., (2008), Enquête Marsouin 2007 : Communes bretonnes et TIC., une fracture numérique entre collectivités locales, octobre, 4 pages de recherche M@ $@$ rsouin.

DAVIS F.D. (1989), Perceived usefulness, perceived ease of use, and user acceptance of information systems research from 1991 to 2001, Information System Journal 14(3), p.197-235.

Decman M., KunStelJ M., Leben A., (2003), " Current state of e-Government in Slovenian municipalities », The Electronic Journal of e-Government, Vol.3, Issue 3, pp. 117-128, available online at www.ejeg.com

DeJEan S., SouQuet A., (2011), Diffusion régionale des politiques publiques d'E-gouvernement, Une étude sur les sites web des communes bretonnes, Document de travail, M@rsouin.

DE LA Coste P. (2003), L'Hyper République: bâtir l'administration en réseau autour du citoyen, janvier, Berger-Levrault, 79 pages.

DiJk J.V., Pieterson W., Deuren (VAn) A., Ebbers W., (2007), « e-Services for citizens: the Dutch usage case », in Wimmer M.A., Scholl H.J., Gronlund A. (eds.): EGOV 2007, LNCS 4656, Springer-Verlag Berlin Heidelberg, p. 155-166.

EbRAhim Z., IRANi Z., AL Shawi S., (2004), « A strategic framework for e-government adoption in public sector organisations », Americas Conference on Information Systems, 2004, New York City, United-States, August.

Fautrero V., Fernandez V., Puel G., (2007), « Les technologies alternatives à l'usage : à propos dune expérimentation satellite / Wifi », Espaces et Sociétés, n¹31, 2007/4, p 137-152.

FINNEY M.M., YoON M.J., (2011), «Interdependence in the technology adoption decision among municipalities », Applied Economics, 43(28), p.4343-4352. Routledge.

FloridA R., 2002, The Rise of the Creative Class: and How It's Transforming Work, Leisure, Community and Everyday Life. New York, Basic Books 
FORnefeld M., Delaunay G., EliXmann D., (2008), The impact of broadband on growth and productivity. A study on behalf of the European Commission, (DG Information Society and media), MICUS.

Fourgous J.M., (2010), Réussir l'école numérique, Rapport mission parlementaire, Documentation Française, Paris

Galliano D., Roux P. (2006), «Les inégalités spatiales dans l'adoption des TIC : le cas des firmes industrielles françaises », Revue Economique, Vol. 57, nº, pp. 1449-1475.

Galliano D., Lethiais V., Soulie N. (2008), «Faible densité des espaces et usages des TIC par les entreprises : besoin d'information ou de communication ? », Revue d'Economie Industrielle, $1^{\mathrm{er}}$ trimestre, Vol.121, pp. 41-64.

GilletT S., LERH W., OSORIO C., (2004), «Local government broadband initiatives », Telecommunications Policy, 28, p.537-558.

Gillett S., Lerh W., OsOrio C., (2006), " Municipal Electric Utilities' Role in Telecommunications services », Telecommunications Policy, Vol. 30, issue 8-9, septembre octobre 2006, p464-480.

Hagerstrand T., (1953), Innovation diffusion as a spatial process, Chicago, University of Chicago Press, 334p., traduction de l'ouvrage pionnier de 1953: Innovationsförloppet ur korologisk synpunkt, Lund, Gleerup.

JACOBS J., (1969), The economy of cities. Random House, New York.

JUN K.N., WEARE C., (2008), The adoption of municipal web sites : on efficiency, power, and legitimacy. Proceedings of the 2008 international conference on Digital government research, Digital Government Society of North America, pp. 272-281.

Katz R., ZenhauSern P., SUTer S., (2008), An evaluation of socio-economic impact of a fiber network in Switzerland, mineo, Polynomics and Telecom Advisory Services, LLC.

KATZ R., SUTER S., (2009), « Estimating the economic impact of the broadband stimulus plan», Columbia Institute for Tele-Information working paper. 
KoutroumPIS P., (2009), « The economic impact of broadband on growth: a simultaneous approach », Telecommunications Policy, 2009, vol. 33, p.471-485. ISSN/0308-5961

Krugman P., (1991), Geography and Trade, MIT Press.

LAYNE K., LEE J., (2001), "Developing fully functional e-Government: a four stage model", Government Information Quarterly, Vol.18, p.122-136.

LAYNE K., LEE J., (2001), « Developing fully functional e-Government: a four stage model », Government Information Quarterly, Vol.18, pp.122-136.

LEHR W.H., OSORIO C.A., GILlETT S.E., (2008), " Measuring broadband's economic impact », Conference on Telecommunications infrastructure and economic performance, Paris, October $16^{\text {th }}-17^{\text {th }}$

LEE J.K., (2003), « A model for monitoring public sector web site strategy », Internet Research, Electronic networking application and policy, vol.13, n 4 , p. 259-266.

LEE C., BerRY F.S., (2011), Testing the development and diffusion of e-Government and eDemocracy: a global perspective, Public Administration Review, (June).

Le Guel F., Penard T., Suire R., (2005), « Adoption et Usage marchand de l'Internet, une étude économétrique sur données françaises », Economie et prévision, n¹67, p. 67-84.

LE Guel F., Penard T., (2004), « Internet et les ménages luxembourgeois : peut-on encore parler de fracture numérique dans le Grand-duché », Economie \& Entreprise, n³ décembre 2004, 21 pages.

Lethias V., Rallet A., Vicente J., (2003), « TIC et réorganisation spatiale des activités économiques : introduction », Revue Géographie, Economie, Société, n 5 , p. 275-285.

LE Mentec M. (2011), «Les fractures numériques sous l'angle des politiques publiques », in Plantard P. et al. (éd), Pour en finir avec la fracture numérique, coll. Usages, Fyp, Limoges, p.4159.

LiMAN MANSAR S., (2006), « e-Government implementation: a comparative evaluation of websites maturity », European and Mediterranean Conference on Information Systems (EMICS), July 6-7 2006, Costa Blanca, Alicante, Spain. 
LONGHI C., (2007), Service, Internet, et développement local : vers une definition des territories numériques, in Mondialisation des services, innovation et dynamiques territoriales, M.C. Monnoyer et P. Ternaux eds, L'Harmattan, Paris 2007.

LONGHi C., RAINELli M., (2010), Poles of competitiveness, a French dangerous obsession? Int. J. Technology Management, Vol. 49, Nos. 1/2/3, 2010, pp. 66-92

LORENTZON S. (1998), « The role of ICT as a locational factor in peripheral region. Example from IT active local authority in Sweden», Netcom, vol.12, n $1,2,3, \mathrm{p} .303-331$.

MARCHANDISE J.F. (2002), «Libérer le "premier kilomètre », le 24/06/02, FING, ACTU.net, http://www.internetactu.net/2002/06/24/librer-le-premier-kilomtre/

MARTin L., Poussing N., (2007), «Le profil des entreprises ayant recours à l'administration électronique : une analyse empirique sur données Luxembourgeoises », Colloque Pré-ICIS'07, UQAM, 7-8 décembre 2007, Montréal.

MARTY F., (2013), «Les partenariats public-privé dans les infrastructures de télécommunications à haut et très haut débit: les enjeux concurrentiels », Revue d'économie industrielle, $\mathrm{n}^{\circ} 141,1^{\mathrm{er}}$ trimestre 2013, p.73-116.

MOON M.J., NORRIS D.F., 2005, « Does managerial orientation matter? The adoption of reinventing government and e-government at the municipal level », Informations Systems Journal, 15(1), p.43-60.

MORGAN K. (1997), « The learning region: institutions, innovation, and regional renewal », Regional Studies, vol. 31.5, p.491-503.

MUSSO P., (2008), Critique de la notion de «territoire numériques », in Quaderni, n66, Printemps 2008. Cyberesp@ce\&territoires, p.15-29.

OCDE, (2004), L’administration électronique, un impératif, Ocde, Paris, 227 pages.

Plantard P., Le Mentec M., Trainoir M., (2011), Pour en finir avec la fracture numérique, Fyp éditions, 167 pages.

Rallet A., Rochelandet F., (2004), «La fracture numérique une faille sans fondements », Réseaux, n¹27-128, 2004/5-6, p.19-54. 
Rallet A., UllmanN C., (2005), «Le "haut débit", nouveau défi du développement local: approches croisées de l'économie et de la géographie », XLIème Colloque de l'ASRDLF, Villes et Territoires face aux défis de la mondialisation, Dijon, 5-7 septembre 2005.

REDDICK C.G., 2004, « A two stage model of e-government growth theories and empirical evidence for US cities », Government Information Quaterly, 21 (2004), p.51-64.

SAINT-AMANT G., (2005), « e-Gouvernement : cadre d'évolution de l'Administration Electronique », Systèmes d'Information et Management, vol.10, n 1 , p. 15-39.

SCHELIN S.H., (2003), « e-Government: an overview », in G.David Garson (ed.) Public Information Technology policy and management issues, pp.120-137, Hershey, PA : Idea Group Publishing.

SIAU K., LONG Y., (2004), « Factors impacting e-Government development », International conference on information systems 2004, Washington DC, Etats-Unis, 11-15 December.

SUIRE R., (2003), « Du cyber district au district lisière : stratégies de localisation des firmes TIC », Géographie, économie et société, n³/4, p. 371-397.

SUIRE R., (2007), «Encastrement social et usages de l'internet : une analyse jointe du commerce et de l'administration électronique », Economie et Prévision, 181, 2007, [3], (B).

Susanto T.D., GoODWIN R., (2010), «Factors influencing citizen adoption of sms-based eGovernment services », Electronic Journal of e-Government, Vol.8, Issue 1, 2010, p.55-71, available online at www.ejeg.com

Trkman P., TURK T., (2009), «A conceptual model for the development of broadband and eGovernment », Government Information Quaterly, 26 (2009), p.416-424.

VAN DER WAAL V., VLOEIMANS J., (2006), « The impact of e-government on the organization: a case study in Amsterdam », Working Papers on Information Systems, Sprouts, p.1-14.

VAn Deursen A., VAn DiJK J., EbBers W., (2006), «Why e-government usage lags behind: explaining the gap between potential and actual usage of electronic public services in the Netherlands", Lecture Notes in Computer Science, 4084, p.269-280. 
ViCENTE J., SUIRE R., (2004), «Observational vs. Interactive learning in locational choice: evidences on ICT clusters formation and stability », Cahiers du GRES 2004-10, Groupement de Recherches Economiques et Sociales.

VICENTE J., (2005), Les espaces de la net-économie : Clusters TIC et aménagement numériques des territoires, Economica, Paris, 148 pages.

VIDAL P., (2007), « La permanence d'une politique publique TIC : De Parthenay « ville numérisée » à Parthenay «ville numérique », Networks and Communication Studies, NETECOM, vol.21 (2007), $n^{\circ} 12$, p.137-164.

WANG Y.S., LIAW Y.W., (2008), Assessing eGovernment system success : a validation of the DeLone and McLean model of information systems success, Government Information Quaterly, 25(4), p.717-733.

Weare C., Musso J.A., Hale M.L., (1999), Electronic Democracy and the diffusion of municipal web pages in California, Administration \& Society, 31(1), p.3-27. 